\title{
Envolvimento macrovascular e esclerose sistêmica
}

\author{
Macrovascular involvement and systemic sclerosis \\ Emmanuelle Tenório Albuquerque Madruga Godoi ${ }^{1}$, Alexandre Domingues Barbosa ${ }^{2}$, \\ Juannicelle Tenório Albuquerque Madruga Godoi ${ }^{3}$, Mariana Atanásio Morais Ramos ${ }^{3}$, \\ Jocelene Tenório Albuquerque Madruga Godoi ${ }^{4}$, Silvio Romero de Barros Marques ${ }^{5}$, \\ Ângela Luzia Branco Pinto Duarte ${ }^{6}$
}

\section{Resumo}

Este artigo tem como objetivo revisar os aspectos descritos na literatura sobre o acometimento da macrovasculatura na esclerose sistêmica e avaliar a ocorrência e distribuição das alterações macrovasculares nos pacientes com esclerose sistêmica através do eco-Doppler e do índice tornozelo-braço, além da associação desses achados com as características demográficas, forma clínica, tempo de evolução da doença, fenômeno de Raynaud (FR), alterações digitais, ulcerações de membros, reabsorção de falange, amputação, bem como fatores de risco e antecedentes da doença ateromatosa. O estudo foi prospectivo, do tipo série de casos, constituído de 20 pacientes, sendo 19 do sexo feminino, com idade média de 46,30 anos. Todos os pacientes tinham fenômeno de Raynaud objetivo, $85 \%$ a forma clínica difusa, $55 \%$ alteração de polpa digital, $15 \%$ úlcera atual de membros, $25 \%$ reabsorção de falange, nenhuma amputação e $70 \%$ de um a quatro fatores de risco. Foram estudadas pelo eco-Doppler as artérias aorta e carótida, dos membros inferiores e superiores, para a avaliação de espessamento do complexo íntimo-medial e presença de placas e aneurismas. Nas artérias dos membros inferiores, foi também realizado o índice tornozelo-braço. O índice tornozelo-braço foi normal em todos os pacientes; entretanto, $12(60 \%)$ destes apresentaram doença macrovascular pelo eco-Doppler, sendo nove $(45 \%)$ na aorta, seis $(30 \%)$ nas carótidas, uma $(5 \%)$ nas artérias dos membros superiores e sete $(35 \%)$ nas dos membros inferiores. Observou-se associação entre doença macrovascular e alterações de polpas digitais $(\mathrm{p}=0,0045)$. A doença macrovascular foi identificada em $60 \%$ dos pacientes através do eco-Doppler, mas não pelo índice tornozelo-braço, que foi normal em todos. Verificou-se associação significante da doença macrovascular com as alterações atuais de polpas digitais, o que não ocorreu com as demais variáveis estudadas.

Palavras-chave: Esclerose sistêmica, efeito Doppler, aterosclerose.

\begin{abstract}
The objectives of this article are to review aspects described in the literature on macrovasculature onset in systemic sclerosis and to assess occurrence and distribution of macrovascular alterations in patients with systemic sclerosis using Doppler ultrasound and ankle-brachial index. In addition, the article evaluates the association of these findings with demographic characteristics, clinical form, clinical course of the disease, Raynaud's phenomenon, digital changes, limb ulcers, reabsorption of phalanges, amputation, and risk factors and antecedents of atheromatous disease. A prospective, case series study of 20 patients was performed. The sample was comprised of 19 women, with a mean age of 46.30 years. All patients had objective Raynaud's phenomenon, $85 \%$ were diffuse, $55 \%$ had digital pulp changes, $15 \%$ current limb ulcer, $25 \%$ reabsorption of phalange, no amputations, and $70 \%$ presented one to four risk factors. Studies were performed by color Doppler ultrasound of the aortic and carotid arteries and lower and upper limb arteries to assess thickening of the intima-media complex, presence of plaques and aneurysms. Ankle-brachial index was also performed in lower limb arteries. Ankle-brachial index was normal in all patients, but $12(60 \%)$ had macrovascular disease assessed by Doppler ultrasound, nine (45\%) of these in the aorta, six (30\%) in the carotid arteries, one (5\%) in the upper limb arteries, and seven $(35 \%)$ in lower limb arteries. There was an association between macrovascular disease and digital pulp changes $(\mathrm{p}=0.0045)$. Macrovascular disease was identified in $60 \%$ of the patients via Doppler ultrasound, but not via ankle-brachial index, which resulted normal in all patients. There was a significant association between macrovascular disease and current digital pulp changes, which did not occur with the other variables.
\end{abstract}

Keywords: Systemic sclerosis, Doppler effect, atherosclerosis.

1. Mestranda, Medicina Interna, Universidade Federal de Pernambuco (UFPE), Recife, PE. Especialista, Angiologia, SBACV.

2. Aluno, Curso de Especialização em Reumatologia, UFPE, Recife, PE.

3. Acadêmica de Medicina, UFPE, Recife, PE.

4. Professora. Doutora associada. Chefe, Departamento de Medicina Clínica, UFPE, Recife, PE.

5. Professor titular, Serviço de Cirurgia Vascular, Hospital de Clínicas, UFPE, Recife, PE.

6. Professora titular. Chefe, Serviço de Reumatologia, Hospital de Clínicas, UFPE, Recife, PE.

Não foram declarados conflitos de interesse associados à publicação deste artigo.

Trabalho realizado no Serviço de Reumatologia, Hospital das Clínicas, Centro de Ciências da Saúde, Universidade Federal de Pernambuco (UFPE), e no Setor de Radiologia do Hospital Agamenon Magalhães, Recife, PE.

Artigo submetido em 09.01.08, aceito em 12.11.08.

J Vasc Bras. 2008;8(1):65-76.

Copyright ( 2009 by Sociedade Brasileira de Angiologia e de Cirurgia Vascular 


\section{Introdução}

A esclerose sistêmica (ES) é uma doença do tecido conjuntivo, pouco frequente e de etiologia desconhecida. Acomete preferencialmente as mulheres, na proporção de 3:1, dos 35 aos 60 anos, sendo infrequente em crianças e em homens com menos de 30 anos $^{1,2}$. No Brasil, ainda não estão disponíveis os dados de incidência e prevalência que estão sendo estudados pelo Grupo de Esclerose Sistêmica do Projeto Pronuclear (GEPRO), implantado em $2003^{3}$.

A ES acomete múltiplos órgãos, incluindo pele, sistema cardiovascular, pulmões, trato gastrointestinal e rins, cujo estudo histopatológico tem demonstrado edema das células endoteliais e/ou hiperplasia intimal da microvasculatura. $\mathrm{O}$ fenômeno de Raynaud (FR) e as ulcerações digitais são exemplos comuns desse tipo de acometimento. Ambas as alterações, estrutural e funcional, são os elementos que contribuem para a vasculopatia na $\mathrm{ES}^{4-6}$.

A ES é subdividida em dois grupos: forma difusa e limitada, sendo a primeira mais grave (com manifestações viscerais e vasculares), e a forma limitada, menos complexa $^{7,8}$.

As alterações vasculares são mais evidentes na forma limitada (FR severo, telangiectasias e um tipo primário de hipertensão pulmonar na doença tardia). Anticorpo anticentrômero é um fator de risco conhecido para isquemia digital e aparece, principalmente, na forma limitada de longa duração $0^{9,10}$.

Embora a doença microvascular seja um dos marcadores da ES, o acometimento da macrocirculação pode ocorrer. Este envolvimento não tem sido largamente reconhecido, embora autores sugiram, em estudos descritivos, uma associação mais que casual ${ }^{11-13}$. O objetivo deste artigo foi revisar os aspectos presentes na literatura sobre o acometimento da macrovasculatura e a descrição da ocorrência e distribuição das alterações macrovasculares nos pacientes com ES do Hospital das Clínicas (HC) da Universidade Federal de Pernambuco (UFPE) através do eco-Doppler e do índice tornozelobraço (ITB).

\section{Doença microvascular na ES}

A disfunção vascular é um elemento fundamental na $\mathrm{ES}^{14,15}$, e um sistema vascular ativado, com o predomínio da vasoconstrição, está frequentemente associado a uma significativa morbidade na evolução da doença ${ }^{16}$.

Infiltrados inflamatórios perivasculares e apoptose endotelial com diminuição da angiogênese têm sido observados nos estágios iniciais da ES; entretanto, os estágios terminais são caracterizados por excessivo acúmulo de proteínas na matriz extracelular da pele e de órgãos internos. Surpreendentemente, a despeito da angiogênese insuficiente, um fator de crescimento endotelial vascular, que tem função de proteger contra úlceras digitais, encontra-se aumentado, porém sua expressão prolongada pode ter efeito paradoxal, determinando formação de vasos irregulares semelhantes aos observados na $\mathrm{ES}^{17,18}$.

O FR é considerado a manifestação mais frequente e precoce da ES, podendo ocorrer em até $95 \%$ dos pacientes ${ }^{19}$. Caracteriza-se por episódios reversíveis de vasoespasmo das extremidades, associados à alteração de coloração típica nas mãos e/ou pés, usualmente após contato com frio ou estresse emocional. Na sua forma primária, esse evento é benigno e sem dano tecidual ${ }^{20}$.

No FR secundário à ES, alterações vasculares características somam-se ao vasoespasmo arterial, podendo acarretar dano tecidual irreversível, como reabsorção da polpa e falange distal, ulcerações, cicatrizes e gangrena de extremidade. Essas lesões vasculares são caracterizadas principalmente por proliferação miointimal, por exuberante atividade de fibroblasto e disfunção endotelial, presentes nas pequenas artérias e microcirculação, nos estágios iniciais da doença ${ }^{21,22}$.

A microangiopatia periférica na ES é bem documentada pela capilaroscopia, que é um método estabelecido para a avaliação do componente morfológico no FR. O padrão conhecido e denominado de escleroderma é observado em 83 a $97 \%$ dos pacientes ${ }^{23}$ e caracterizado pela presença de dilatação (ectasia) e distorção capilar associada a áreas de completa desvascularização, além da diminuição difusa da quantidade de alças ${ }^{22}$. Esse padrão não é exclusivo da ES, podendo também ser encontrado em pacientes com dermatomiosite e doença mista do tecido conjuntivo. O laser Doppler tem sido 
utilizado como nova técnica de quantificação de fluxo na microcirculação de pacientes com $\mathrm{ES}^{24}$.

Tradicionalmente, o envolvimento vascular na ES tem sido predominantemente microvascular, associado ao desequilíbrio entre vasoconstrição e vasodilatação, com predomínio da primeira ${ }^{15}$. Apesar de o FR ser a manifestação vascular mais frequente, estudos recentes também vêm evidenciando crescente acometimento macrovascular em pacientes esclerodérmicos ${ }^{4,5,25,26}$. A detecção do acometimento vascular é elemento fundamental na ES, pois está frequentemente associada à significativa morbimortalidade na evolução da doença ${ }^{27,28}$.

Uma redução importante na complacência arterial dos pacientes esclerodérmicos está relacionada à elasticidade do vaso, podendo ser consequência das alterações do tecido conectivo e refletir no aumento do risco cardiovascular $^{19,29}$.

\section{Doença macrovascular}

A aterosclerose, principal causa de doença macrovascular (DMV), tem sido associada à ES. Sua identificação precoce tem influência na redução da morbimortalidade desses pacientes ${ }^{26}$.

É uma doença sistêmica caracterizada pela formação de placas de colesterol no nível da camada íntima. Todas as artérias podem ser acometidas, mas as consequências clínicas são mais importantes no comprometimento das coronárias, das carótidas e das artérias dos membros inferiores (MMII). A doença arterial obstrutiva crônica constitui, ao lado da insuficiência coronariana e do acidente vascular cerebral isquêmico, uma das três manifestações clínicas do mesmo processo fisiopatológico: a aterotrombose ${ }^{30}$.

A doença arterial periférica (DAP), de origem ateromatosa, é mais frequente nos MMII e em homens com idade entre 50 e 70 anos, embora venha sendo diagnosticada em indivíduos mais jovens. Sua prevalência é variável de acordo com o método diagnóstico utilizado e a população estudada ${ }^{31}$.

A claudicação intermitente (CI) dos MMII é a manifestação clínica característica da DAP, sendo consequência do processo isquêmico tecidual. A claudicação acontece quando a necessidade metabólica do tecido é maior que a recebida pelo aporte sanguíneo ${ }^{32,33}$. A dor de repouso surge quando a chegada do sangue não é suficiente nem para manter o metabolismo basal. A rede de colateral, que reduz os sintomas, desenvolve-se com exercícios de caminhada ${ }^{32}$. Estudos evidenciaram CI mais prevalente em pacientes com ES do que na população geral ${ }^{26,34}$. Na classificação da DAP, proposta por Fontaine em 1954, a CI corresponde ao estádio II $^{32,35}$.

Os fatores de risco para a DAP ateromatosa são os mesmos da aterosclerose, em especial o tabagismo, a hipertensão arterial sistêmica (HAS), o diabetes melito (DM) e a dislipidemia ${ }^{30,36}$.

\section{Aterosclerose e ES}

Alteração da macrovasculatura associada à dislipidemia tem sido descrita em doenças do colágeno. No lúpus eritematoso sistêmico (LES), a presença dos autoanticorpos contra lipoproteína lipase (anti-LPL), enzima que hidrolisa triglicerídeos, determina elevação dos níveis séricos dos mesmos e da aterosclerose precoce. A presença do anti-LPL IgG em pacientes com ES está associada à níveis elevados de triglicerídeos, fibrose cutânea mais extensa, fibrose pulmonar, envolvimento cardíaco e antitopoisomerase $\mathrm{I}^{37}$.

Lippi et al. ${ }^{38}$, em estudos preliminares sobre o perfil lipídico de pacientes com ES, evidenciaram que a lipoproteína-a (Lpa) pode agir em sinergismo com outras condições pró-trombóticas conhecidas na patogênese de uma variedade de alterações vasculares. Tais alterações predispõem ao desenvolvimento de complicações trombóticas, salientando-se a importância da identificação e eventual tratamento desse subgrupo de pacientes.

\section{Métodos diagnósticos da DMV}

Uma anamnese adequada pode ser responsável pelo diagnóstico da DAP em 90\% dos casos, por isso questionários como o de Edinburgh e o da Organização Mundial de Saúde (OMS) têm um alto valor preditivo positivo $^{36,39,40}$.

O ITB é um método simples, não invasivo, de alto valor preditivo para a DAP e tem associação significante com o risco de mortalidade cardiovascular. Esse índice também pode ser usado como um bom indicador de DAP em pacientes com ES ${ }^{10,26,41}$. Divide-se a pressão sistólica mais alta de cada tornozelo pela maior pressão sistólica braquial ${ }^{42}$. Dessa forma, o ITB é igual à 
Tabela 1 - Interpretação dos valores do índice tornozelo-braço na arteriopatia de membros inferiores

\begin{tabular}{lc}
\hline ITB distal & Interpretação \\
\hline$>1,3$ & Artérias incompressíveis \\
0,9 e 1,3 & Estado hemodinamicamente normal \\
0,75 e 0,9 & AMI bem compensada \\
0,4 e 0,75 & AMI medianamente compensada \\
$<0,4$ & AMI com comprometimento severo
\end{tabular}

AMI = arteriopatia de membros inferiores; ITB = índice tornozelo-braço.

Fonte: Leger \& Boccalon ${ }^{35}$.

maior pressão sistólica no tornozelo dividida pela maior pressão sistólica braquial.

O valor normal do ITB varia de 0,9 a 1,3 , sendo este associado ao estado clínico da arteriopatia de MMII $(\mathrm{AMI})^{35}$ (Tabela 1). A redução do ITB indica acometimento nas artérias dos MMII, e valores menores ou iguais a 0,9 estão associados a um significativo aumento do risco cardiovascular, particularmente infarto do miocárdio e acidente vascular cerebral (AVC), independente de outros fatores de risco ${ }^{41}$.

O teste da esteira consiste em caminhar numa esteira elétrica e pode ser realizado pela maioria dos arteriopatas. Anota-se o perímetro de marcha no início da claudicação e o perímetro máximo onde ocorre a parada pela dor. O ITB é medido antes e imediatamente quando ele para de caminhar. Em um indivíduo normal, o ITB aumenta com o esforço, e em um paciente com DAP, diminui. Essa diminuição é diretamente proporcional ao grau da lesão arterial e inversamente ao grau de compensação pela rede colateral ${ }^{35,36,43}$.

O eco-Doppler constitui método não-invasivo, de alta resolução, que permite avaliação detalhada da macrovasculatura e combina três componentes: o modo-B, o Doppler pulsado e o modo color.

O modo-B (Figura 1A) fornece imagem bidimensional em tempo real, sendo usado para a avaliação anatômica do vaso, permitindo localizá-lo nos tecidos moles, medir calibre e visualizar irregularidades (placas ou trombos) na luz do mesmo. O Doppler pulsado (Figura 1B) permite a avaliação da velocidade do fluxo. Pode-se quantificar a velocidade e avaliar o padrão de fluxo, que varia de acordo com o tipo de artéria, sendo de baixa ou alta resistência. O modo color (Figura 1C) permite que os dados da velocidade sejam determinados sob a forma de um mapa, com cores codificadas superpostas à imagem da ultrassonografia modo-B. A cor da imagem baseia-se na direção do fluxo ${ }^{44-46}$.

O espessamento do complexo íntimo-medial é um marcador não-invasivo e precoce de alteração da parede arterial, facilmente medido na artéria carótida, usando-se o modo-B da ultrassonografia. Sua utilização em pesquisa é crescente, e a medição pode ser feita de duas maneiras: medida manual em múltiplos sítios da carótida extracraniana, nas paredes anterior e/ou posterior, e medida por programa de aquisição automática, na parede posterior do segmento distal da artéria carótida comum. $\mathrm{O}$ espessamento pode refletir no aumento do risco cardiovascular global. A avaliação epidemiológica dos dados indica que um aumento do complexo íntimo-medial (maior que $1 \mathrm{~mm}$ ) é aceito como espessamento e pode representar risco maior de infarto do miocárdio e/ou doença cerebrovascular ${ }^{46-48}$.

A arteriografia convencional e digital é um método invasivo, considerado padrão-ouro para o diagnóstico da DMV, pois permite a construção de um mapa arterial detalhado e fidedigno. Por existirem métodos menos invasivos de diagnóstico, seu uso preferencial é para o tratamento de lesões arteriais ${ }^{49}$.

A angiorressonância magnética (ARM) realça o fluxo sanguíneo, pois suprime os sinais gerados por tecidos estáticos e amplia sinais do sangue circulante. Depois da obtenção de várias imagens isoladas, existe uma somação ou recombinação dos dados para se obter imagem angiográfica convencional. A ARM apresenta como 


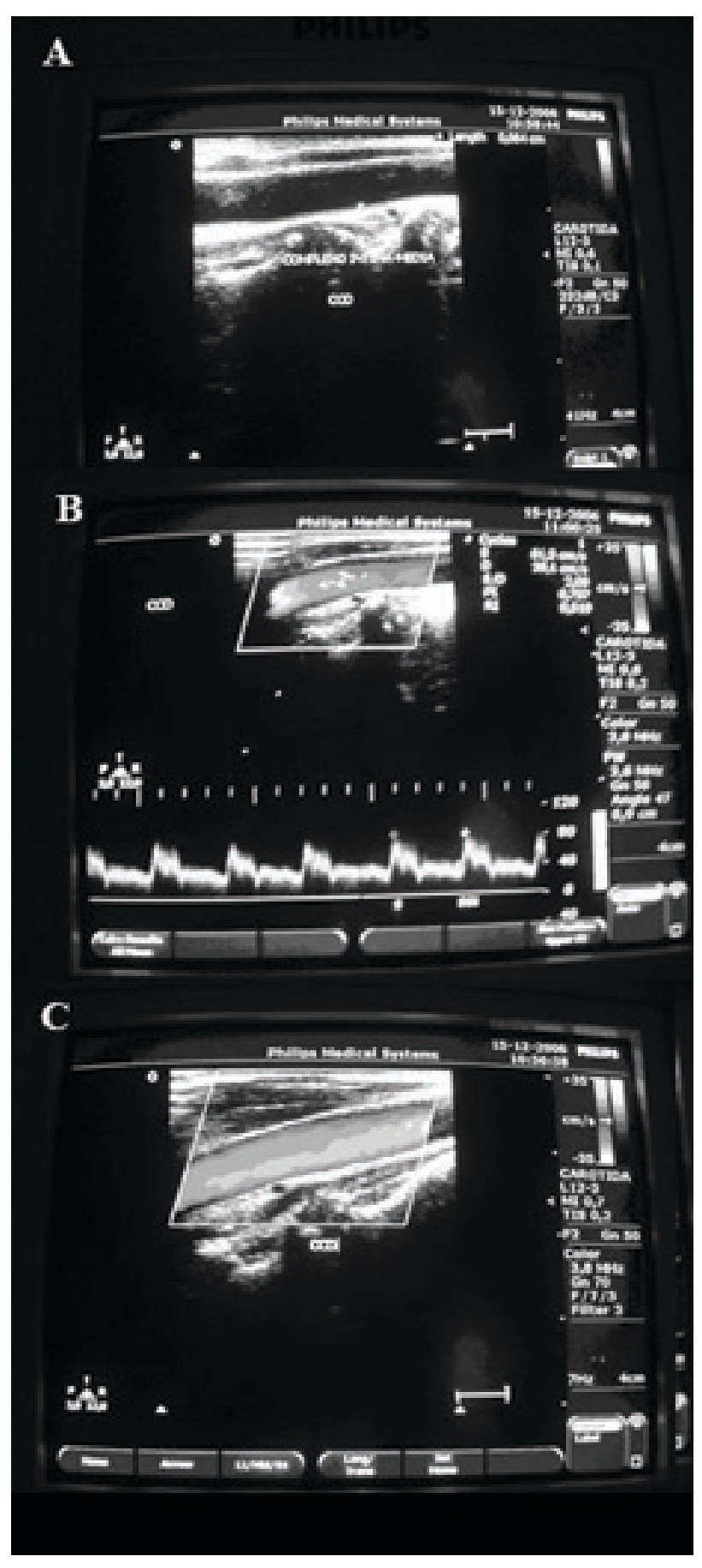

Figura 1 - Eco-Doppler: A) modo-B; B) modo pulsado; C) modo color

vantagens: não ser invasiva, não envolver exposição à radiação ionizante e poder ser realizada sem uso de meio de contraste - embora para melhor definição e menor tempo de exame seja feita com o gadolínio, meio de contraste intravenoso com mínimos efeitos colaterais ${ }^{50}$.
A TC helicoidal (ou espiral) ampliou as indicações da TC na avaliação dos vasos. Esta técnica foi ainda mais aprimorada com o desenvolvimento da TC de múltiplos detectores multi-slice, que é um método não-invasivo e de grande valor para a investigação das patologias vasculares. Tem como desvantagens o uso de meio de contraste intravenoso iodado e a utilização de radiação ionizante, sendo a dose habitualmente utilizada superior à da TC helicoidal. Pela presença de múltiplos detectores, variando de 6 a 64, permite uma alta resolução temporal e espacial, um estudo amplo em poucos segundos e uma varredura desde a aorta até as artérias mais distais $^{51}$.

\section{DMV e ES}

A ES é caracterizada por lesão fibrótica complexa do tecido conjuntivo e alterações do leito vascular, incluindo proliferação da camada intimal e espessamento fibroso da camada média. Alguns possíveis mecanismos são responsáveis pela isquemia, incluindo vasoespasmo, dano endotelial e anormalidades da homeostase. A extensão do envolvimento vascular tem sido mais bem definida, não só em nível dos órgãos internos como na periferia ${ }^{16,52}$.

$\mathrm{Na}$ ES, o padrão característico da microvasculatura inclui disfunção endotelial e distúrbios de coagulação. Alterações similares também têm sido observadas em pacientes com DMV secundária à aterosclerose. Esses achados patogênicos podem sugerir associação de aterosclerose e $\mathrm{ES}^{26}$.

A DMV na ES tem sido descrita em relatos de casos e estudos retrospectivos pós-morte, refletindo a falta de identificação desse padrão e o desconhecimento de sua prevalência ${ }^{34}$. Ultimamente, alterações da macrovasculatura associada à dislipidemia têm sido descritas ${ }^{37,38}$.

Youssef et al. ${ }^{53}$ estudaram pacientes que tinham DMV severa de membros superiores (MMSS) e mínimos fatores de risco cardiovascular. Em uma amostra comprometida da artéria ulnar de um paciente, foi identificado importante estreitamento luminal por material acelular, sem nenhuma evidência de ateroma.

Em um estudo com 53 pacientes esclerodérmicos, Veale et al. ${ }^{34}$ utilizaram o questionário de CI da OMS para determinar a prevalência de DMV sintomática na 
ES. A CI foi encontrada em $10(21,7 \%)$ pacientes, sendo dois da forma difusa e oito da CREST (Calcinose, Raynaud, hipomotilidade Esofageana, Esclerodactilia, Telangiectasia). Comparando a população desse estudo com a população estudada em Edinburgh, onde a CI foi de $4,6 \%$, Veale et al. ${ }^{34}$ observaram uma maior prevalência de CI na população com ES. Como fatores de risco, foram identificados: HAS em quatro pacientes, tabagismo em sete e hipercolesterolemia em dois; nenhum deles era diabético nem fazia uso de corticosteroide. Ainda nesse estudo, 70\% dos claudicantes esclerodérmicos apresentavam doença cardíaca isquêmica sintomática, com angina ou infarto agudo do miocárdio atual ou prévio, quando comparados com 19,4\% dos sem CI.

Em um estudo de coorte retrospectivo no período de 1974-90, Youssef et al. ${ }^{4}$ avaliaram a prevalência de DMV nos MMSS em mulheres com ES, com duração mínima de 5 anos de doença, comparadas com o grupo-controle que não apresentava ES. A DAP ocorreu em 18 (58\%) pacientes e em apenas três $(9,6 \%)$ controles (risco relativo $=6,0$ ), estando associada com severa morbidade. $\mathrm{O}$ estudo histológico das pacientes com DAP sugeriu espessamento intimal discreto na artéria ulnar.

Stafford et al. ${ }^{25}$, em estudo de coorte retrospectivo, com 20 pacientes esclerodérmicos e 20 controles, analisaram os resultados de Doppler de MMII, das artérias do pescoço e do abdômen, correlacionando-os com fatores de risco para aterosclerose (HAS, hiperlipidemia, tabagismo e DM). A CI foi mais frequente nos pacientes esclerodérmicos. Doença arterial ulnar ocorreu em 10/19 (53\%) dos pacientes, sendo bilateral em 6/10 (60\%). A ausência de associação entre ES e doença arterial de MMII foi outro achado desse estudo. Os autores ressaltaram que doença arterial ulnar tem repercussão importante na evolução da reversibilidade ou irreversibilidade da isquemia digital.

Ho et al. ${ }^{26}$, em estudo de caso-controle com 54 pacientes esclerodérmicos e 43 controles, avaliaram a prevalência de DMV. Todos foram submetidos a uma triagem convencional para fatores de risco da aterosclerose. Para a identificação da DMV, foram utilizados Doppler carotídeo e ITB. Não houve diferença significante em relação aos fatores de risco entre os grupos. Cerca de $64 \%$ dos pacientes tiveram doença carotídea quando comparados a $35 \%$ do controle $(\mathrm{p}=0,007)$. Nove (17\%) dos pacientes tiveram ITB menor que 0,9 , comparados com nenhum do controle $(p=0,003)$. Não foram observadas correlações entre o acometimento macrovascular e o tempo de doença ou a forma clínica da $\mathrm{ES}^{26}$.

Através da angiografia de 29 pacientes, sendo 14 com acroesclerose e 15 com esclerose ascendente proximal, Stucker et al. ${ }^{54}$ encontraram estenose das artérias dos MMSS em 27/29 (93\%). A frequência da oclusão arterial diminuiu de $90 \%$ das artérias digitais para $31 \%$ da artéria ulnar e nenhum comprometimento da artéria radial. A intensidade do FR não se correlacionou com a dos achados angiográficos; entretanto, estes se correlacionaram com a extensão da esclerose cutânea e com os achados sorológicos.

Wan et al. ${ }^{10}$ avaliaram retrospectivamente a medida do ITB em 119 pacientes esclerodérmicos, sendo 91 com a forma limitada e $28 \mathrm{com}$ a difusa. O ITB foi medido de forma habitual, e o valor normal de referência foi igual a 1,0. O ITB foi inferior a 1,0 em 14 pacientes $(12 \%), 11$ com a forma limitada e três com a difusa.

No período de 1992 a 2000, Dick et al. ${ }^{55}$ avaliaram 26 de 1.400 pacientes com ES submetidos à arteriografia ou angioplastia, devido a isquemia crítica de membros, levando em consideração idade, sexo e fatores de risco cardiovascular (tabagismo, HAS, DM e hipercolesterolemia). A angiografia dos MMSS demonstrou doença distal isolada em $86 \%$ dos pacientes com ou sem fatores de risco cardiovascular, tais como o tabagismo. Nos MMII, houve uma associação altamente significante entre os fatores de risco e DMV e, inversamente, entre ausência desses fatores e doença distal (abaixo do joelho). Os autores concluíram que a indicação da angiografia nos MMSS independe dos fatores de risco, enquanto que, para os MMII, a presença destes faz-se necessária.

Em estudo retrospectivo, avaliando 15 pacientes esclerodérmicos, com FR intenso, caracterizado por úlceras digitais refratárias, teste de Allen positivo e doença arterial ulnar oclusiva por angiografia, Taylor et al. ${ }^{5}$ encontraram oclusão em todos, sendo bilateral 
em nove pacientes. Oito deles foram submetidos à revascularização, e o exame histológico da amostra da artéria ulnar revelou fibrose da camada íntima, estreitamento do lúmen, camada muscular ligeiramente hipertrofiada e adventícia normal, consistentes com os achados histológicos da doença microvascular, sugerindo que a vasculopatia não é aterosclerótica.

Cheng et al. ${ }^{19}$ realizaram estudo sobre as propriedades biomecânicas (elástica e muscular) e espessamento do complexo íntimo-medial das artérias carótida e femoral em 19 pacientes com ES difusa, 33 na forma limitada e 21 controles. Observaram progressiva e significante redução $(\mathrm{p}<0,001)$ na propriedade elástica da artéria carótida dos pacientes esclerodérmicos com a forma limitada em relação ao grupo-controle.

Em estudo de caso-controle com 45 pacientes esclerodérmicos, avaliados por ultra-sonografia para espessamento do complexo íntimo-medial das artérias carótidas e identificação de fatores de risco (HAS, obesidade, DM, tabagismo, dislipidemia), Beyne-Rauzy et al. ${ }^{56}$ não encontraram diferença significante em relação a esses fatores. Ainda com esse mesmo grupo de pacientes, Leger et al. ${ }^{57}$ encontraram anormalidades arteriais em 23/45 (51,1\%) através do eco-Doppler, sendo 14/23 (61\%) nos MMSS, 3/23 (13\%) nos MMII e 6/23 (26\%) em ambos. Nestes dois estudos, do mesmo centro, parece ser a doença carotídea menos frequente e a DMV periférica dos membros mais frequente no grupo esclerodérmico.

Em estudo seccional de 221 pacientes com doença arterial sintomática de MMII (CI em 93\%), foram avaliados: aspectos sociodemográficos, fatores de risco para aterosclerose, FR, outra doença arterial e procedimento cirúrgico vascular. Diferença significante foi observada nos pacientes com FR e doença coronariana (6/8, 75\%) quando comparados com controles $(64 / 179,35,8 \%)$. Olmedo et al. ${ }^{58}$ concluíram que, em pacientes ateroscleróticos de alta morbimortalidade, a presença de FR determina cinco vezes mais doença coronariana e, surpreendentemente, ES foi mais frequente que o esperado, sugerindo relação bidirecional entre ES e aterosclerose.

Hasegawa et al. ${ }^{59}$ avaliaram a macrovasculatura em oito pacientes esclerodérmicos com úlceras digitais e gangrena através de arteriografia dos MMSS e MMII, correlacionando-as com perfil de autoanticorpos e fatores de risco cardiovascular. Envolvimento macrovascular foi detectado em $7 / 8(87,5 \%)$ dos pacientes esclerodérmicos, e os autores concluíram que o envolvimento macrovascular, detectado com arteriografia, não é raro em pacientes com ES e ulcerações digitais ou gangrena.

\section{Métodos}

Para ilustrar este tema, foi realizado no HC da UFPE estudo prospectivo, tipo série de casos, constituído de 20 pacientes, sendo 19 do sexo feminino, com idade média de 46,30 anos. O estudo foi aprovado no comitê de ética em pesquisa envolvendo seres humanos do Centro de Ciências da Saúde da UFPE e teve como objetivo avaliar a ocorrência e distribuição das alterações macrovasculares em uma população com ES através do ecoDoppler e do ITB. Foi também estudada a associação desses achados com as características demográficas, forma clínica, tempo de evolução da doença, FR, alterações digitais, ulcerações de membros, reabsorção de falange e amputação, bem como fatores de risco e antecedentes da doença ateromatosa. A forma clínica difusa esteve presente em $85 \%$ dos pacientes. Todos tinham FR, $55 \%$ apresentaram alteração de polpas digitais, $15 \%$, úlcera atual de membros, $25 \%$, reabsorção de falange, e não houve amputação (Tabela 2).

\section{Resultados}

Fatores de risco para aterosclerose - um a quatro fatores - estavam presentes em $70 \%$ da população estudada. A aorta, carótidas, bem como as artérias dos MMSS e MMII foram estudadas por eco-Doppler para a avaliação de espessamento do complexo íntimomedial, presença de placas e aneurismas (Tabela 3). Nas artérias dos MMII, foi também realizado o ITB. Como resultados, o ITB foi normal em todos os pacientes; entretanto, $12(60 \%)$ destes apresentaram DMV, sendo nove $(45 \%)$ na aorta, sete (35\%) nas artérias dos MMII, seis $(30 \%)$ nas carótidas e um $(5 \%)$ nas artérias dos MMSS. Observou-se associação entre DMV e alterações de polpas digitais $(\mathrm{p}=0,0045)$ (Tabela 4). A ecoDoppler identificou DMV em $60 \%$ dos pacientes com ES. Nenhum paciente apresentou ITB anormal. 
Tabela 2 - Distribuição das alterações de polpas digitais, de úlceras de membros inferiores e/ou superiores, reabsorção de falange e amputação nos pacientes com esclerose sistêmica

\begin{tabular}{lc}
\hline Variável & $\mathbf{n}(\mathbf{\%})$ \\
\hline Alterações de polpas digitais* & $10(50,0)$ \\
Atual & $5(25,0)$ \\
Pregressa & $3(15,0)$ \\
Reabsorção de falange (atual/pregressa) & $1(5,0)$ \\
Atual & $5(25,0)$ \\
Pregressa & 0 \\
Reabsorção de falange (atual/pregressa) & 0 \\
Amputação & \\
\hline
\end{tabular}

* Considerando que um mesmo paciente poderia apresentar mais de uma alteração digital, registra-se apenas a base para o cálculo dos percentuais e não o total.

Verificou-se associação significante da DMV com as alterações atuais de polpas digitais, e não houve associação da DMV com os fatores de risco (Tabela 5).

\section{Discussão}

Em nosso estudo, a DMV, avaliada pelo eco-Doppler, esteve presente em $60 \%$ da população, o que não ocorreu com o ITB, que foi normal em todos os pacientes. Youssef et al ${ }^{4}$ identificaram a DMV em 58\% dos pacientes com ES, utilizando a combinação da eco-Doppler, arteriografia, história de CI e alterações dos pulsos. A DMV foi identificada em apenas $10 \%$ do grupo-controle deste estudo. Leger et al. ${ }^{57}$, por meio da eco-Doppler e do ITB, encontraram anormalidades arteriais periféricas em $51,1 \%$ dos esclerodérmicos e $6,7 \%$ dos pacientes do grupo-controle.
Ho et al. ${ }^{26}$ identificaram alterações do ITB em 17\% dos pacientes com ES estudados e em nenhum do grupocontrole. Wan et al. ${ }^{10}$ também encontraram alteração desse índice em 14 (12\%) pacientes; entretanto, esses autores definiram como anormais valores inferiores a 1,0. Caso tivéssemos utilizado essa mesma referência, $15 \%$ dos nossos pacientes teriam o ITB alterado. Optamos pelo ponto de corte inferior a 0,9 (sensibilidade de $95 \%$ e especificidade de $100 \%$ quando comparados à arteriografia, padrão-ouro de diagnóstico) por ter sido adotado pela maioria dos estudos ${ }^{26,35,41}$.

Não evidenciamos alterações nas artérias ulnares. Stafford et al. ${ }^{25}$ evidenciaram a DMV por acometimento das artérias ulnares em 53\% dos esclerodérmicos, sendo bilateral em $60 \%$. Taylor et al. ${ }^{5}$ identificaram

Tabela 3 - Distribuição do tipo e localização das alterações na aorta, carótidas e artérias dos membros superiores e inferiores de pacientes com esclerose sistêmica por eco-Doppler

\begin{tabular}{lccc}
\hline Artérias & $\begin{array}{c}\text { Espessamento } \\
\mathbf{n}(\%)\end{array}$ & $\begin{array}{c}\text { Placa } \\
\mathbf{n}(\%)\end{array}$ & $\begin{array}{c}\text { Aneurisma } \\
\mathbf{n}(\%)\end{array}$ \\
\hline Aorta & $8(40)$ & $5(25)$ & $1(5)$ \\
Carótidas & $4(20)$ & $4(20)$ & 0 \\
Artérias dos MMSS & $1(5)$ & 0 & 0 \\
Artérias dos MMII & $4(20)$ & $4(20)$ & 0 \\
\hline
\end{tabular}

MMII = membros inferiores; MMSS = membros superiores. 
Tabela 4 - Associação da doença macrovascular segundo a ocorrência e tipo de alterações das polpas digitais em pacientes com esclerose sistêmica

\begin{tabular}{lcccc}
\hline \multirow{2}{*}{$\begin{array}{l}\text { Alterações das polpas } \\
\text { digitais }\end{array}$} & \multicolumn{3}{c}{ Doença macrovascular } & \\
\cline { 2 - 4 } Alteração & Sim, $\mathbf{n}(\%)$ & Não, $\mathbf{n}(\%)$ & Total, $\mathbf{n}(\%)$ & $\mathbf{p}^{*}$ \\
Atual & $10(90,9)$ & $1(9,1)$ & $11(100,0)$ & $\mathrm{p}=0,0045^{\dagger}$ \\
Pregressa & $9(90,0)$ & $1(10,0)$ & $10(100,0)$ & $\mathrm{p}=0,0198^{\dagger}$ \\
\hline
\end{tabular}

* Teste exato de Fisher.

${ }^{\dagger}$ Associação significante ao nível de $5 \%$.

Tabela 5 - Associação da doença macrovascular segundo os fatores de risco da doença ateromatosa, isolados ou agrupados em pacientes com esclerose sistêmica

\begin{tabular}{lcccc}
\hline & \multicolumn{3}{c}{ Doença macrovascular } & \multirow{2}{*}{ Fator risco } \\
\cline { 2 - 3 } & Sim, $\mathbf{n}(\%)$ & Não, $\mathbf{n}(\%)$ & Total, $\mathbf{n}$ (\%) & \\
\hline HAS & $5(71,4)$ & $2(28,6)$ & $7(100,0)$ & $\mathrm{p}=0,6424$ \\
Diabetes melito & 0 & $1(100,0)$ & $1(100,0)$ & $\mathrm{p}=0,40000$ \\
Hipercolesterolemia & $5(83,3)$ & $1(16,7)$ & $6(100,0)$ & $\mathrm{p}=0,3246$ \\
Hipertrigliceridemia & $3(60,0)$ & $2(40,0)$ & $5(100,0)$ & $\mathrm{p}=1,0000$ \\
Tabagismo (atual/ & $6(87,5)$ & $1(14,3)$ & $7(100,0)$ & $\mathrm{p}=0,1577$ \\
pregresso) & & & \\
\hline
\end{tabular}

HAS = hipertensão arterial sistêmica.

* Teste exato de Fisher.

oclusão da artéria ulnar em 15 pacientes esclerodérmicos, sendo bilateral em nove. Leger et al. ${ }^{57}$ encontraram a DMV em pacientes com ES, sendo 31\% acometidos nos MMSS e $6 \%$ nos MMII, resultados inversos aos descritos em nosso estudo. Vale ressaltar que o acometimento das artérias dos MMSS foi mínimo neste estudo, ao contrário dos achados de outros autores ${ }^{5,58}$, que acreditam ser esse envolvimento um marcador da DMV na ES. As artérias ulnares foram estudadas por ultrassonografia e apresentaram fluxos preservados e paredes conservadas. Achados segmentares e lesões digitais podem não ter sido identificados por não termos realizado estudo arteriográfico.

Não houve associação significante da DMV com idade, sexo, raça, tempo de evolução e forma clínica da ES, além do FR e dos fatores de risco para doença ateromatosa. Wan et al. ${ }^{10}$ e Ho et al. ${ }^{26}$ também não encontraram associação de DMV com as formas clínicas limitada e difusa, que apresentaram a mesma prevalência.

Em nossa casuística, não houve associação entre a DMV e os fatores de risco para a doença ateromatosa, nem agrupados nem isolados. Stafford et al..$^{25}$ evidenciaram associação da DMV com o tabagismo e a HAS. Wan et al. ${ }^{10}$ demonstraram a associação do tabagismo com a diminuição do ITB.

Em nosso estudo, houve associação significante entre a DMV e alterações de polpas digitais, aspecto não descrito na literatura pesquisada. O envolvimento macrovascular pode somar-se às alterações microvasculares próprias da ES, exacerbando os distúrbios hemodinâmicos distais existentes, responsáveis pelas alterações das polpas digitais.

Um aspecto a ser ressaltado é o fato de que a DAP é predominantemente ateromatosa e mais prevalente em 
homens idosos. A nossa população foi, sobretudo, de mulheres dos 30 aos 59 anos (exceto por uma paciente de 73 anos) e sem associação significante com fatores de risco para a doença ateromatosa. Esses achados sugerem que a DMV por nós evidenciada seja decorrente da ES.

Vale salientar que a DMV da nossa população foi menos significante clinicamente do que em outros estu$\operatorname{dos}^{54,55}$, uma vez que nenhum dos nossos pacientes era claudicante, tinha alteração do ITB ou apresentava comprometimento significante das artérias por meio da eco-Doppler.

O acometimento macrovascular é responsável pelo aumento da morbidade nos pacientes esclerodérmicos. A associação da ES com dislipidemia tem sido descrita e sugere relação entre a mesma e a doença aterosclerótica, apesar de histologicamente não se evidenciar a clássica lesão ateromatosa. Por serem os sintomas da DMV semelhantes aos dos portadores de doença ateromatosa, o conhecimento desta última permite um diagnóstico precoce, um tratamento adequado e uma melhoria na qualidade de vida dos pacientes esclerodérmicos com esse tipo de envolvimento.

\section{Conclusão}

A DMV foi identificada em $60 \%$ dos pacientes através da eco-Doppler, mas não pelo ITB, que foi normal em todos. Verificou-se associação significante da DMV com as alterações atuais de polpas digitais, o que não ocorreu com as demais variáveis estudadas.

\section{Referências}

1. Hummers LK, Wigley FM. Scleroderma. In: Imboden Y, Hellmann DB, Stone JH. Current rheumatology: diagnosis and treatment. San Francisco: McGraw Hill; 2004. p. 189-97.

2. Collier DH. Esclerose sistêmica. In: West SG. Segredos em reumatologia. Porto Alegre: Artmed; 2000. p. 160-8.

3. Sampaio-Barros PD, Andrade LEC. GEPRO: do sonho à realidade. Rev Bras Reumatol. 2004;44:11-2.

4. Youssef P, Brana T, Englert H, Bertouch J. Limited scleroderma is associated with increased prevalence of macrovascular disease. J Rheumatol. 1995;22:469-72.

5. Taylor MH, McFadden JA, Bolster MB, Silver RM. Ulnar artery involvement in systemic sclerosis (scleroderma). J Rheumatol. 2002;29:102-6.

6. Duque FLV, Mello NA. Trombogênese - Trombofilia. J Vasc Bras. 2003;2:105-18.
7. Masi AT, Rodnan GP, Medsger Jr TA, et al. Preliminary criteria for the classification of systemic sclerosis (scleroderma). Arthritis Rheum. 1980;23:581-90.

8. Leroy EC, Black C, Fleischmajer R, et al. Scleroderma (systemic sclerosis): classification, subsets and pathogenesis. J Rheumatol. 1988;15:202-5.

9. Campbell PM, Leroy EC. Pathogenesis of systemic sclerosis: a vascular hypotesis. Semin Arthritis Rheum. 1975;4:351-68.

10. Wan MC, Moore T, Hollis S, Herrick AL. Ankle brachial pressure index in systemic sclerosis: influence of disease subtype and anticentromere antibody. Rheumatology (Oxford). 2001;40:1102-5.

11. Furey NL, Schmid FR, Kwaan HC, Friederici HH. Arterial thrombosis in scleroderma. Br J Dermatol. 1975;93:683-93.

12. Dorevitch MI, Clemens LE, Webb JB. Lower limb amputation secondary to large vessel involvement in scleroderma. Br J Rheumatol. 1988;27:403-6.

13. Sella EMC, Bazílio AP, Yoshinari NH. Oclusão de grandes vasos em esclerodermia: relato de caso. Rev Bras Reumatol. 1998;38:245-8.

14. LeRoy EC. Systemic sclerosis: a vascular perspective. Rheum Dis Clin North Am. 1996;22:675-94.

15. Herrick AL. Vascular function in systemic sclerosis. Curr Opin Rheumatol. 2000;12:527-33.

16. Kahaleh MB, LeRoy EC. Autoimmunity and vascular involvement in systemic sclerosis (SSc). Autoimmunity. 1999;31:195-214.

17. Distler JH, Kalden JR, Gray S Distler O. [Vascular changes in the pathogenesis of systemic sclerosis]. Z Rheumatol. 2004;63:446-50.

18. Distler JH, Gay S, Distler O. Angiogenesis and vasculogenesis in systemic sclerosis. Rheumatology. 2006;45(Suppl 3):iii26-7.

19. Cheng KS, Tiwari A, Boutin A, et al. Carotid and femoral arterial wall mechanics in scleroderma. Rheumatology (Oxford). 2003;42:1299-305.

20. Leroy EC, Medsger TA Jr. Raynaud's phenomenon: a proposal for classification. Clin Exp Rheumatol. 1992;10:485-8.

21. Prescott RJ, Freemont AJ, Jones CJ, Hoyland J, Fielding P. Sequential dermal microvascular and perivascular changes in the development of scleroderma. J Pathol. 1992;166:255-63.

22. Kayser C, Andrade LEC. Ausência de correlação entre as alterações morfológicas e bioquímicas na microcirculação de pacientes com esclerose sistêmica. Rev Bras Reumatol. 2004;44:12-8.

23. Bredemeier M, Xavier RM, Capobianco KG, et al. Capilaroscopia periungueal pode sugerir atividade de doença pulmonar na esclerose sistêmica. Rev Bras Reumatol. 2004;44:19-30.

24. Clark S, Campbell F, Moore T, Jayson MI, King TA, Herrick AL. Laser Doppler imaging: a new technique for quantifying microcirculatory flow in patients with primary Raynaud's phenomenon and systemic sclerosis. Microvasc Res. 1999;57:284-91.

25. Stafford L, Englert H, Gover SJ, Bertouch J. Distribution of macrovascular disease in scleroderma. Ann Rheum Dis. 1998;57:476-9. 
26. Ho M, Veale D, Eastmond C, Nuki G, Belch J. Macrovascular disease in systemic sclerosis. Ann Rheum Dis. 2000;59:39-43.

27. Sampaio-Barros PD, Samara AM, Marques Neto JF. Estudo sobre as diferentes formas clínicas e escores cutâneos na esclerose sistêmica. Rev Bras Reumatol. 2004;44:1-7.

28. Soares CJG, Cruz BA. Severe digital ischemia due to systemic sclerosis successfully treated with bosentan: case report. J Vasc Bras. 2007;6:276-9.

29. Glasser SP, Arnett DK, Mc Veigh GE, et al. Vascular compliance and cardiovascular disease: a risk factor or a marker? Am J Hypertension. 1997;10:1175-89.

30. Dedola M, Godoi E, Coppe G, et al. Risk factors management in 5708 ambulatory patients suffering from peripheral vascular disease followed in urban practices. Arch Mal Coeur Vaiss. 2005;98:1177-8.

31. Marchand G. Epidémiologie et facteurs de risque de l'artériopathie oblitérante des membres inférieurs. Ann Cardiol Angéiol. 2000;150:119-27.

32. Maffei FHA, Lastória S, Yoshida WB, et al. Diagnóstico clínico das doenças arteriais periféricas. In: Maffei FHA, Lastória S, Yoshida WB, Rollo HA, Giannini M, Moura R. Doenças vasculares periféricas. $3^{\text {a }}$ ed. Rio de Janeiro: Medsi; 2002. p. 287-305.

33. Olin JW. Management of patients with intermittent claudication. Int J Clin Pract. 2002;5:687-93.

34. Veale DJ, Collidge TA, Belch JJ. Increased prevalence of symptomatic macrovascular disease in systemic scleorsis. Ann Rheum Dis. 1995; 54:853-5.

35. Leger P, Boccalon H. Bilan d'une artériopathie des members inférieurs (AMI). In: Boccalon H. Guide pratique des maladies vasculaires. $2^{\mathrm{a}}$ ed. France: Masson; 2001. p. 13-38.

36. Dormandy JA, Rutherford RB. Management of peripheral arterial disease (PAD). TASC Working Group. TransAtlantic Inter-Society Consensus (TASC). J Vasc Surg. 2000;31(1 Pt 2):S1-296.

37. Kodera M, Hayakawa I, Komura K, et al. Anti-lipoprotein lipase antibody in systemic sclerosis: association with elevated serum triglyceride concentrations. J Rheumatol. 2005;32:629-36.

38. Lippi G, Caramaschi P, Montagnana M, Salvagno GL, Volpe A, Guidi G. Lipoprotein(a) and the lipid profile in patients with systemic sclerosis. Clin Chim Acta. 2006;364:345-8. Epub 2005 Aug 19.

39. Fowkes FG, Housley E, Cawood EH, Macintyre CC, Ruckley CV, Prescott RJ. Edinburgh artery study: prevalence of asymptomatic and symptomatic peripheral arterial disease in the general population. Int J Epidemiol. 1991;20:384-92.

40. Rose GA. The diagnosis of ischaemic heart pain and intermittent claudication in field surveys. Bull World Health Organ. 1962;27:645-58.

41. Spácil J, Spácabilová J. The ankle-brachial blood pressure index as a risk indicator of generalized atherosclerosis. Semin Vasc Med. 2002;2:441-5.

42. Atsma F, Bartelink M-LEL, Grobbee DE, et al. Best reproducibility of the ankle-arm index was calculated using Doppler and dividing highest ankle pressure by highest arm pressure. J Clin Epidemiol. 2005;58:1282-8.
43. Feinberg RL, Gregory RT, Wheeler JR, et al. The ischemic window: A method for the objective quantitation of the training effect in exercise therapy for intermittent claudication. J Vasc Surg. 1992;16:244-50.

44. Salles-Cunha SX, Morais Filho D. Princípios básicos. In: Engelhorn CA, Morais Filho D, Barros FS, Coelho N. Guia prático de ultra-sonografia vascular. Rio de Janeiro: Dilivros; 2007. p. 1-15.

45. Hood DB, Brewer DJ. Eco Color Doppler dos membros. In: Eton D. Doença vascular: abordagem multidisciplinar ao diagnóstico e tratamento. $2^{\mathrm{a}}$ ed. Rio de Janeiro: Dilivros; 2003. p. 546-53.

46. Barros FS, Pontes SM. Doença carotídea aterosclerótica. In: Engelhorn CA, Morais Filho D, Barros FS, Coelho N. Guia prático de ultra-sonografia vascular. Rio de Janeiro: Dilivros; 2007. p. 17-37.

47. Bots ML, Evans GW, Riley WA, Grobbee DE. Carotid intima-media thickness measurements in intervention studies: design options, progression rates, and sample size considerations: a point of view. Stroke. 2003;34:2985-94. Epub 2003 Nov 13

48. Simon A, Gariepy J, Chiron G, Megnien JL, Levenson J. Intima-media thickness: a new tool for diagnosis and treatment of cardiovascular risk. J Hypertension. 2002;20:159-69.

49. Moura R. Angiografia por subtração digital. In: Maffei FHA, Lastória S, Yoshida WB, Rollo HA. Doenças vasculares periféricas. $3^{\text {a }}$ ed. Rio de Janeiro: Medsi; 2002. vol. 1, cap. 28, p. $384-98$

50. Aizenstein RI, Shamim D, Wilbur AC. Angioressonância magnética e angiotomografia computadorizada: cabeça e pescoço In: Eton D. Doença vascular: abordagem multidisciplinar ao diagnóstico e tratamento. $2^{\mathrm{a}}$ ed. Rio de Janeiro: Dilivros; 2003. p. 125-43.

51. Chiou P, Anderson CM. Computed tomography, angiography and magnetic resonance angiography of the extracranial carotid arteries In: Higgins CB, Ross A. MRI and CT of the cardiovascular system. 2nd ed. Philadelphia: Lippincott Williams \& Wilkins; 2005. p. 538-60.

52. Marie I. Pathogenesis of scleroderma. Rev Prat. 2002;52:1873-81

53. Youssef PP, Englert H, Bertouch J. Large vessel occlusive disease in CREST and scleroderma. Ann Rheum Dis. 1993;52:464-6.

54. Stücker M, Quinna S, Memmel U, et al. Macroangiopathie of the upper extremities in progressive systemic sclerosis. Eur $\mathbf{J}$ Med Res. 2000;5:295-302.

55. Dick EA, Aviv R, Francis I, et al. Catheter angiography and angioplasty in patients with scleroderma. Br J Radiol. 2001;74:1091-6.

56. Beyne-Rauzy O, Leger P, Godel A, et al. Intima-media thickness evaluation in 45 systemic sclerosis compared to health subjects matched for sex and gender [abstract]. In: The American College of Rheumatology National Scientific Meetings, 2004.

57. Leger P, Beyne-Rauzy O, Arista S, et al. Macrovascular disease assessment in systemic sclerosis: results of a prospective case-control study [abstract]. In: The American College of Rheumatology National Scientific Meetings, 2004. 
58. Olmedo JFJ, Rial R, Fuentes M, Morata C, López R, Serrano J. Raynaud's phenomenon, systemic sclerosis, and macrovascular peripheral disease [abstract]. In: The Annual European Congress of Rheumatology, Amsterdan, 2006.

59. Hasegawa M, Nagai Y, Tamura A, et al. Arteriographic evaluation of vascular changes of the extremities in patients with systemic sclerosis. Br J Dermatol. 2006;155:1159-64.
Correspondência:

Dra. Emmanuelle Tenório A. M. Godoi

Rua Marquês de Tamandaré, 162/1402, Poço da Panela CEP 52061-170 - Recife, PE

E-mail: godoiemmanuelle@hotmail.com

\section{Aviso aos ex-residentes}

Se você foi residente até 2008 e ainda não está inscrito em sua Regional, procure regularizar sua situação para passar a receber o J Vasc Bras imediatamente, sem qualquer ônus. Ajude a manter o cadastro da SBACV atualizado. 